\title{
Présentation de la Société suisse de médecine esthétique
}

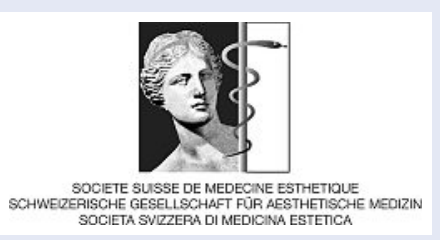

\section{Xavier Martina \\ Michel Pfulg ${ }^{b}$}

a PD Dr méd. Président de la SSME

b Dr méd.,

Vice-président de la SSME

Nous remercions le Docteur Francesco Failla pour la version italienne de ce texte que vous trouverez sur www.saez.ch $\rightarrow$ Numéro actuel ou $\rightarrow$ Archives $\rightarrow 2010 \rightarrow 32$ ainsi que sur www.ssme.ch

Correspondance: PD Dr méd. Xavier Martin Président de la SSME 64, avenue de Rumine CH-1005 Lausanne

xmartin@worldcom.ch

www.ssme.ch
Dès le début des années 70, certains ophtalmologues, comme Alan Scott [1] à San Francisco puis Alfred Huber [2] à Zurich, ont commencé à agrandir leur arsenal thérapeutique en ajoutant aux techniques chirurgicales l'usage de la toxine botulinique, en particulier pour le traitement de certains strabismes et dans certaines pathologies palpébrales. Ces dernières pathologies réunissant souvent des problèmes fonctionnels et esthétiques, le traitement de l'un n'allait pas sans l'autre. Et, c'est ainsi que certains ophtalmologues comme Jean Carruthers [3], qui fut le fellow d'Alan Scott, auxquels se sont bientôt joints des médecins d'autres spécialités, ont commencé à forger les outils de la médecine esthétique.

La Société suisse de médecine esthétique (SSME), fondée en 1994, est un forum, lieu de rencontres et d'échanges, puisque tous les tissus et/ou organes participent de près ou de loin à l'esthétique de l'être et de son corps. La SSME réunit des spécialistes de nombreuses disciplines médicales lors de son Congrès annuel.

Dans le cadre de son congrès annuel, la SSME organise des cours pratiques donnés par des spécialistes reconnus. Exceptionnellement le Congrès 2010 de la SSME a eu lieu en même temps que celui de la Société Belge de médecine esthétique qui organisait le Congrès européen de médecine esthétique à Bruxelles. La délégation suisse à ce Congrès a de loin été la plus importante délégation étrangère.

En plus de son Congrès annuel, la SSME organise deux séminaires de formation pratique par an. Les derniers séminaires ayant eu lieu à Berne, à Genève, à Fribourg, à Nyon, à Sion et à Montreux.

Bien que n'ayant pas pour mission d'arbitrer des conflits entre patients et praticiens, la SSME peut être amenée à donner des recommandations susceptibles de désamorcer des conflits.

Afin que les médecins suisses pratiquant la médecine esthétique puissent remplir leurs obligations légales (art. 40 let. B LPMéd), la SSME a introduit en 2008 un contrôle de la formation permanente de ses membres, comparable à celui des autres spécialités et calqué sur les règles de la FMH. La SSME encourage les médecins pratiquant la médecine esthétique en Suisse à adhérer à cette société.

Nos grands voisins, France, Allemagne et Italie offrent différents curriculums de formation en médecine esthétique dont certains sont de haut niveau. Nous encourageons nos membres et nos futurs membres à suivre ces formations que la Suisse ne peut pas offrir.
Nous sommes à disposition pour renseigner les médecins intéressés par ces formations postgraduées.

La SSME vient aussi d'introduire un Registre des complications en médecine esthétique. Les modalités concernant ce registre se trouvent sur www.ssme.ch.

Du point de vue de sa taille, la SSME compte 60 membres, étant ainsi comparable à la Société suisse de neurochirurgie qui en compte environ 90.

La SSME est membre de l'Union Internationale de Médecine Esthétique (UIME), organisme réunissant la majorité des Sociétés de médecine esthétique de la planète, dont la Turquie avec ses 50 membres et la Chine avec ses 10000 membres.

L'UIME organise chaque année des Congrès internationaux, comme le Congrès européen qui a lieu en alternance avec le Congrès international. Le dernier congrès international organisé conjointement par l'UIME et la SSME fut le congrès européen qui a eu lieu en 2004 à Zurich.

La SSME est une association dont les buts principaux, énoncés dans ses statuts sont:

- Réunir des médecins qui travaillent dans le domaine de la médecine esthétique en Suisse;

- Avancement de la médecine esthétique dans les domaines scientifique et pratique et planification de son développement futur en Suisse;

- Maintien des relations avec les disciplines voisines;

- Maintien de relations confraternelles entre ses membres;

- Défense de l'éthique dans l'exercice de la médecine esthétique.

Ces informations sont rassemblées sur le site internet de la société, www.ssme.ch

La médecine esthétique est avant tout une discipline médicale et la SSME travaille dans le but que les critères de qualité et d'éthique soient respectés et contrôlés.

\section{Références}

1 Scott AB, Rosenbaum A, Collins CC. Pharmacologic weakening of extraocular muscles. Invest Ophthalmol. $1973 ; 12: 924-7$

2 Huber A, Meyer M. Anwendung von Botulinumtoxin in der Ophthalmologie. Klin Mbl Augenheilk. 1986; 188:89-94.

3 Carruthers JD, Carruthers JA. Treatment of glabellar frown lines with C. botulinum-A exotoxin. J Dermatol Surg Oncol. 1992;18:17-21. 


\section{Presentazione della Società Svizzera di medicina Estetica}

Dall'inizio degli anni 70, certi oftalmologi, come Alan Scott (1) a San Francisco e poi Alfred Huber (2) a Zurigo, hanno cominciato ad espandere il loro arsenale terapeutico aggiungendovi alle tecniche chirurgiche usuali l'applicazione della tossina botulinica, in particolare per il trattamento di certe forme di strabismo ed in alcuni casi di patologie palpebrali. Queste ultime patologie racchiudono sovente degli aspetti funzionali ed estetici, e la cura degli uni non va senza la cura degli altri, ed è così che alcuni oftalmologi come Jean Carruthers (3), che era il discepolo di Alan Scott, al quale si sono poi aggiunti medici di altre specialità, hanno cominciato a creare gli strumenti della medicina estetica.

Visto che tutti $i$ tessuti e/o organi partecipano da vicino o da lontano all'estetica dell'essere e del suo corpo, la società svizzera di medicina estetica (SSME), fondata nel 1994, è diventata un forum e luogo di incontri e di scambi interdisciplinari. La SSME riunisce gli specialisti di diverse discipline mediche in occasione del suo congresso annuale.

Nell'ambito del suo congresso annuale, la SSME organizza dei corsi pratici istruiti da specialisti riconosciuti. Eccezionalmente il congresso del 2010 della SSME si è svolto contemporaneamente a quello della società Belga di medicina estetica che organizzava il congresso europeo a Bruxelles. La delegazione Svizzera a questo congresso è stata sicuramente la più importante delegazione straniera.

Oltre al suo congresso annuale, la SSME organizza due seminari di formazione pratica all'anno. Gli ultimi seminari hanno avuto luogo a Berna, Ginevra, Friborgo, Nyon, Sion ed a Montreux.

La SSME tratta pure domande o eventuali denunce fatte da pazienti o da medici.

In modo che i medici svizzeri praticanti la medicina estetica possano adempiere ai loro obblighi legali (art. 40 lett. B LPMed) la SSME ha introdotto nel 2008 un controllo della formazione permanente dei suoi membri, paragonabile a quella delle altre specialità $\mathrm{e}$ basate sulle regole della $\mathrm{FMH}$.

La SSME incoraggia quindi i medici praticanti la medicina estetica in Svizzera ad aderire a questa società.

I paesi nostri vicini, Francia, Germania ed Italia, offrono differenti curriculum di formazione in medicina estetica di cui alcuni sono di livello molto alto. Noi incoraggiamo i nostri membri ed i membri futuri a seguire queste formazioni che la Svizzera non può offrire. Siamo a disposizione per informare i medici interessati da queste formazioni postgraduate.

La SSME ha pure introdotto un registro delle complicazioni in medicina estetica. Le modalità concernenti questo registro si trovano sul sito www.ssme.ch. 
Dal punto di vista della sua grandezza, la SSME conta 60 membri, paragonabile quindi alla Società svizzera di neurochirurgia che ne conta ca. 90.

La SSME è membro dell'Unione Internazionale di Medicina Estetica (UIME), organismo che raggruppa la maggior parte delle Società di medicina estetica del pianeta, dalla Turchia con i suoi 50 membri, alla Cina con i suoi 10'000 membri!

L'UIME organizza ogni anno dei congressi internazionali come il congresso europeo che ha luogo in alternanza con il congresso internazionale. L'ultimo congresso internazionale organizzato congiuntamente tra la UIME e la SSME è stato il congresso europeo che si è svolto nel 2004 a Zurigo.

La SSME è un associazione i cui scopi principali, enunciati negli statuti sono:

- Riunire i medici che la lavorano nell'ambito della medicina estetica in Svizzera.

- Promuovere la medicina estetica nell'ambito scientifico e pratico e pianificare il suo sviluppo futuro in Svizzera.

- Mantenere delle relazioni con le discipline vicine.

- Mantenere le relazioni collegiali tra i suoi membri.

- Difendere l'etica nell'esercizio della medicina estetica.

Queste informazioni sono riunite sul sito internet della società: www.ssme.ch.

La medicina estetica è prima di tutto una disciplina medica e la SSME lavora con lo scopo di far rispettare e di controllare i criteri di qualità e di etica.

\section{Referenze:}

1) Scott $A B$, Rosenbaum $A$, Collins CC. Pharmacologic weakening of extraocular muscles. Invest Ophthalmol. 12: 924-7; 1973.

2) Huber A, Meyer M. Anwendung von Botulinum toxin in der Ophtalmologie. Klin Mbl Augenheilk 188: 89-94; 1986.

3) Carruthers JD, Carruthers JA. Treatment of glabellar frown lines with C. botulinum-A exotoxin. J Dermatol Surg Oncol. 18:17-21; 1992. 